\title{
Terahertz two-cylinder waveguide coupler for transverse-magnetic and transverse-electric mode operation
}

\author{
M. Theuer, ${ }^{1,2}$ A. J. Shutler, ${ }^{1}$ S. Sree Harsha, ${ }^{1}$ R. Beigang, ${ }^{2}$ and D. Grischkowsky ${ }^{1, a)}$ \\ ${ }^{1}$ School of Electrical and Computer Engineering, Oklahoma State University, Stillwater, \\ Oklahoma 74078, USA \\ ${ }^{2}$ Department of Physics and Research Center OPTIMAS, University of Kaiserslautern, \\ 67663 Kaiserslautern, Germany
}

(Received 10 January 2011; accepted 24 January 2011; published online 18 February 2011)

\begin{abstract}
We report the coupling and guiding of broadband terahertz radiation using a two-cylinder waveguide coupler. For the transverse electromagnetic TEM $\left(\mathrm{TM}_{0}\right)$ geometry, the two opposing metal cylinders exhibit an amplitude transmission comparable to that of the cylindrical silicon lens coupled parallel-plate waveguide, but in the transverse-electric orientation the two-cylinder coupler shows much better amplitude transmission. (C) 2011 American Institute of Physics.
\end{abstract}

[doi:10.1063/1.3554761]

Various experimental methods have been developed to investigate crystalline samples in the terahertz $(\mathrm{THz})$ range (far-infrared). Most commonly, these methods have involved samples of either compressed pellets, relatively large single crystals, or more recently thin microcrystalline films on a waveguide plate. ${ }^{1}$ The latter have the important advantage that the crystal quality of such small crystals is excellent and that only a very small amount of sample (typically $100 \mu \mathrm{g}$ ) is needed to obtain strong absorption line signatures. This is due to the lateral compression of the terahertz wave, creating high spatial overlap and strong interaction with the waveguide-contained sample.

Although only a recent development, ${ }^{1,2}$ the use of the parallel plate waveguide (PPWG) in THz-TDS (time-domain spectroscopy) is gaining momentum due to the desirable features of strong sample interaction (high sensitivity) and sharp line narrowing. The use of cylindrical silicon lenses enables the coupling of the terahertz beam into the PPWG with subwavelength spacing, as shown in Fig. 1(a). The confinement of the terahertz beam within this relatively small gap causes a strong interaction with the microcrystalline sample placed within the gap. With single-mode transverse electromagnetic (TEM) propagation (linear polarization of the electric field perpendicular to the metal surface and the direction of propagation), spectral linewidths as small as $7 \mathrm{GHz}$ have been achieved. ${ }^{3}$ The silicon lenses perform well, with an amplitude transmission of typically $20 \%$ through the entire assembly as shown in Fig. 1(a). However, this transmission could be improved by two times, if the Fresnel reflection losses (50\% amplitude loss) due to the two silicon lenses could be avoided. In addition, the small terahertz echoes due to double pass reflection from the lenses could be eliminated. This small delayed echo determines the maximum duration of the transmitted terahertz pulse measurement, whose inverse gives the frequency resolution of the THz-TDS measurement. These improvements would involve replacing the alignment-critical lenses with another coupling scheme.

A recently demonstrated method of coupling the terahertz beam with a wavelength dependent spot size $(9 \mathrm{~mm}$ diameter at $1 \mathrm{THz}$ ) into the PPWG gap without the use of

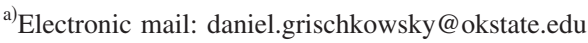

lenses is to use metal flares, ${ }^{4-6}$ somewhat resembling a microwave horn antenna. The efficiency of this microwave-type of coupling is comparable to the quasioptic approach using silicon lenses, due to the fact that the changes of beam extent are introduced smoothly (adiabatically) with respect to the wavelength, by the flares. ${ }^{6,7}$ The more adiabatic the transition, the better the coupling. Amplitude coupling ratios as high as $50 \%$ could be achieved by the slightly curved $12 \mathrm{~cm}$ long metal flares. 5

Here, we propose and demonstrate a significant simplification and standardization of the mechanically flexible flares. ${ }^{5,6}$ Figure 1(b) shows the cross-section of the twocylinder waveguide (WG) coupler, which can be easily constructed by cutting a chord from a full aluminum cylinder. The dimensions of the cylinder pieces were $9.5 \mathrm{~mm}$ (height) with a radius of curvature of $32 \mathrm{~mm}$, giving a chord of 45 $\mathrm{mm}$ length. The particular geometry was chosen to limit the size of the coupler to fit into a cryostat. The two identical pieces are attached together to form a sandwich structure, and spacers are placed at the center edges of the coupler to define different gap widths without truncating the beam.

Operation of the cylinder WG coupler in a single-mode regime is desired for spectroscopic applications, requiring the insertion of geometrically matching sections of PPWG at the indicated midpoint of the coupler. This will require each chord of the coupler to be precisely cut in half and finished to allow the insertion of a matched PPWG plate.

For this demonstration of the single-mode properties of the coupler, single-mode operation can be achieved by adjusting the coupler gap to tune the cut-off frequency, for the next higher order mode, above the highest desired frequency. For an air-filled PPWG, the $\mathrm{m}_{\text {th }}$ order mode has a cut-off
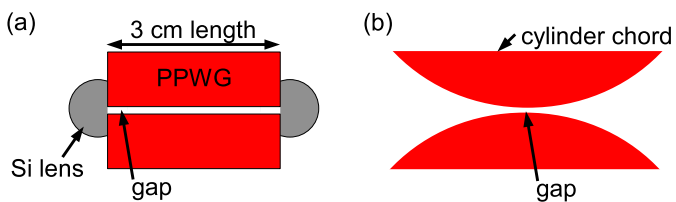

FIG. 1. (Color online) Cross-sections of (a) standard PPWG with cylindrical silicon lenses for coupling. (b) two-cylinder waveguide coupler for which adiabatic wave compression is used for coupling through the subwavelength gap. 


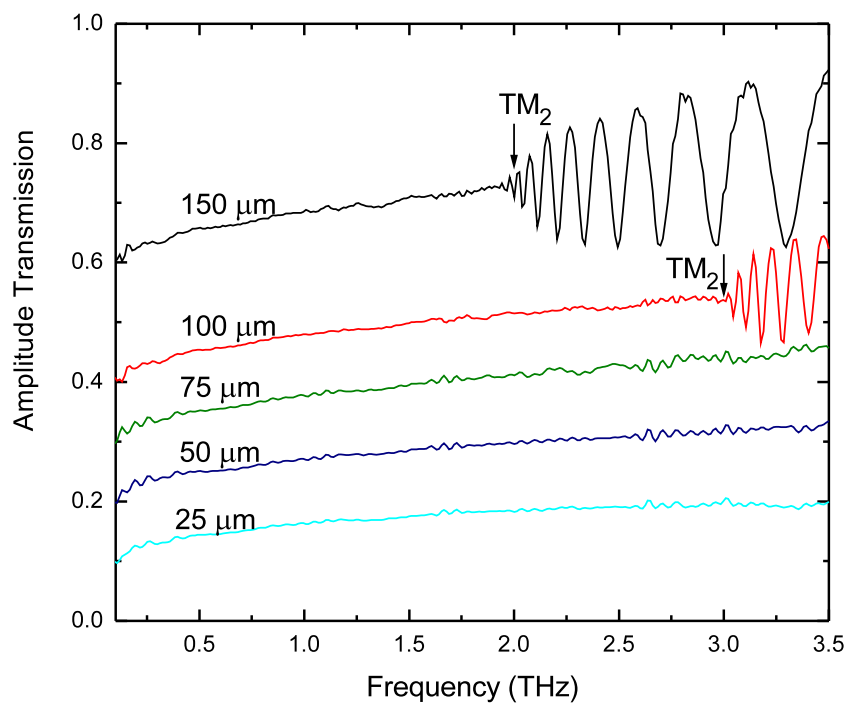

FIG. 2. (Color online) Amplitude transmission of the two-cylinder coupler with TM-polarization for different gap widths. For clarity some of the curves have been lifted as follows: $50 \mu \mathrm{m}$ curve $+0.1,75 \mu \mathrm{m}$ curve +0.2 , $100 \mu \mathrm{m}$ curve +0.3 , and $150 \mu \mathrm{m}$ curve +0.5 . For small gaps single TEMmode propagation is observed, while for larger gaps $\mathrm{TM}_{2}$ propagation also occurs above the indicated cut-off frequencies.

frequency given by $f_{c}=m c /(2 d)$, where $d$ is the gap width. For the transverse-magnetic (TM) case the well known TEM-mode $\left(\mathrm{TM}_{0}\right)$ has no cut-off frequency. This mode is excited if the PPWG is oriented for an incoming terahertz beam polarization perpendicular to the metal plates (see Fig. 1). All of these considerations hold for the two-cylinder WG coupler, even though the effective propagation length associated with the coupler gap is only $1 \mathrm{~mm}$. For the situation shown in Fig. 1(b), the propagation length from the center of the coupler for which the gap separation increases by $2 \mu \mathrm{m}$ is $0.25 \mathrm{~mm}$. Consequently, for a $25 \mu \mathrm{m}$ gap, within a propagation path length of $0.5 \mathrm{~mm}$ centered on the coupler midpoint, the actual separation is between 25 and $27 \mu \mathrm{m}$. Correspondingly, the propagation length from the center of the gap for which the gap separation increases by $8 \mu \mathrm{m}$ is 0.51 $\mathrm{mm}$, and for a $100 \mu \mathrm{m}$ gap, within the propagation length of $1.0 \mathrm{~mm}$ centered on the coupler midpoint, the actual separation is between 100 and $108 \mu \mathrm{m}$.

Results obtained with the two-cylinder WG coupler for the terahertz beam polarization perpendicular to the metal surfaces are shown in Fig. 2 for different gap widths. As the incident Gaussian terahertz beam is symmetric with respect to the beam center (and the center of the coupler), only even TM-mode patterns can be coupled. So in addition to the TEM-mode $\left(\mathrm{TM}_{0}\right)$, the $\mathrm{TM}_{2}$ and the $\mathrm{TM}_{4}$ modes can be excited for frequencies higher than their cut-off frequencies. For a gap of $100 \mu \mathrm{m}$ the onset of the $\mathrm{TM}_{2}$ mode starting at $3 \mathrm{THz}$ can be seen in Fig. 2. If the gap is less than $75 \mu \mathrm{m}$, the two-cylinder coupler is single TEM-mode for frequencies between 0 and $4 \mathrm{THz}$.

The amplitude transmission through the entire coupler for gaps larger than $50 \mu \mathrm{m}$ is approximately $18 \%$, and reduces to $16 \%$ for a gap width of $25 \mu \mathrm{m}$. It was expected that the two-cylinder coupler would show a reduced transmission compared to the larger flares ${ }^{6}$ because the coupling is not as adiabatic, ${ }^{7}$ and a fraction of the incoming terahertz radiation is reflected back to the emitter, reducing the transmission. Even so, the two-cylinder coupler offers good performance

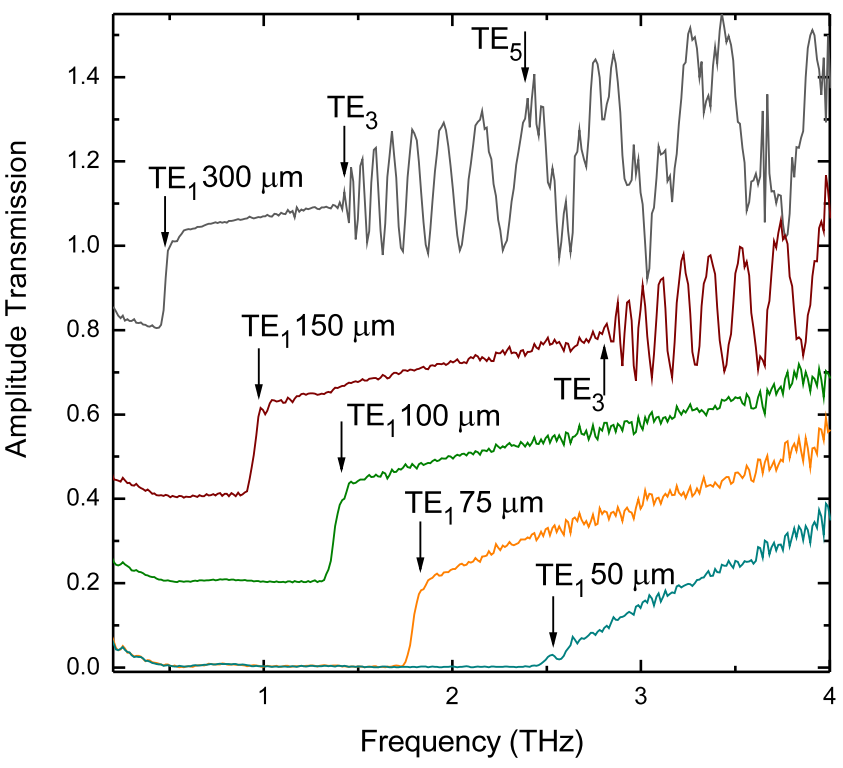

FIG. 3. (Color online) Amplitude transmission of the two-cylinder coupler for TE-polarization and different gap widths. For clarity the curves have been lifted as follows: $100 \mu \mathrm{m}$ curve $+0.2,150 \mu \mathrm{m}$ curve +0.4 , and $300 \mu \mathrm{m}$ curve +0.8 . The transmission turns on sharply at the cut-off frequencies. Also higher TE-modes are observed.

for a given maximum allowed coupler size. For the smaller gaps the amplitude transmission does not drop as much as for the silicon lens coupled PPWGs. Increasing to higher frequencies, the coupling roughly remains constant. This is due to the interplay between the better coupling (smaller pump spot size for higher frequencies) and increasing ohmic losses.

The transverse-electric (TE) modes have attracted recent interest in sensing applications ${ }^{8,9}$ because of their very low ohmic losses and useful field distribution. ${ }^{10} \mathrm{~A}$ major drawback is that the first propagating mode $\mathrm{TE}_{1}$ has a cut-off frequency. This would appear to limit the minimum usable plate separation and reduce the spectral working window between the cut-offs of the first two propagating modes $\mathrm{TE}_{1}$ and $\mathrm{TE}_{3}$. However, recently, there have been several reports of developments and applications based on the propagation of the lowest order $\mathrm{TE}_{1}$ mode. These applications have relied on the use of large gap sizes to mitigate the effects of the cut-off and to improve the throughput. ${ }^{8,10}$

The TE amplitude transmission obtained with the twocylinder coupler is shown in Fig. 3. The steep cut-off of the $\mathrm{TE}_{1}$ mode can be clearly seen for each gap width. For a gap width of $150 \mu \mathrm{m}$ the coupler is multimode due to the turn-on of the $\mathrm{TE}_{3}$ mode. For example, working with a large gap of $300 \mu \mathrm{m}$, the transmission starts above the cut-off frequency of $0.5 \mathrm{THz}$, but becomes multimode above the 1.5 $\mathrm{THz}$ cut-off frequency of the $\mathrm{TE}_{3}$ mode, allowing for a single-mode measurement in the frequency interval between 0.5-1.5 THz. On the other hand, a gap width of $100 \mu \mathrm{m}$ shows $\mathrm{TE}_{1}$ single-mode behavior with a transmission above $1.35 \mathrm{THz}$ (corresponding to an effective gap width of $110 \mu \mathrm{m})$.

For the TE case, the amplitude transmission gets better with higher frequencies, reaching up to $50 \%$. This is caused by the decreasing attenuation coefficient, a unique property of this mode. ${ }^{10}$ Also the initial terahertz spot size is smaller for higher frequencies, which improves the coupling as the 


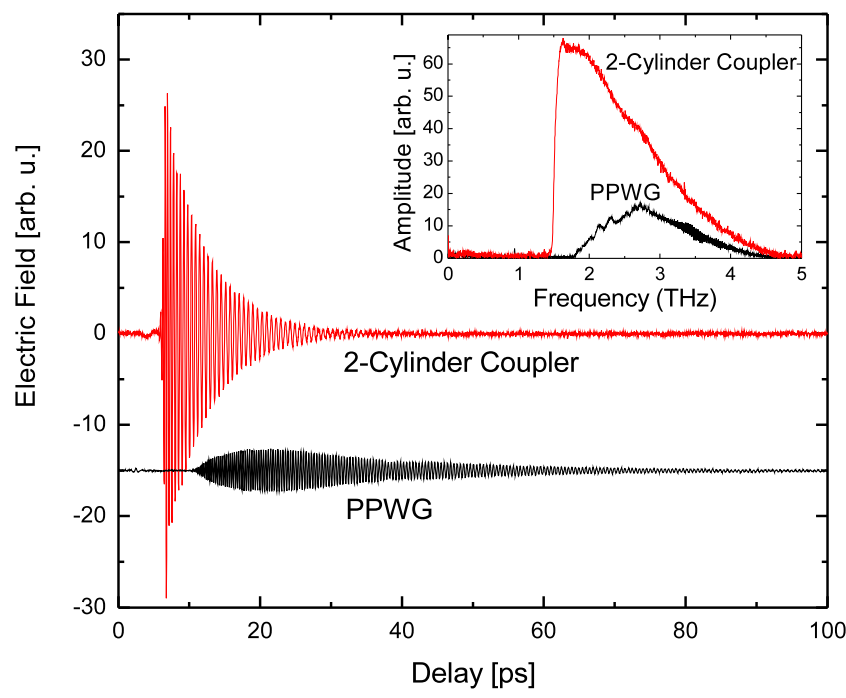

FIG. 4. (Color online) Comparison of the transmitted terahertz pulses for the silicon lens coupled PPWG (lower trace) and the two-cylinder coupler (upper trace), both with $100 \mu \mathrm{m}$ gaps and $\mathrm{TE}_{1}$ mode propagation. Inset: corresponding spectral amplitudes.

intensity is centered more closely to the symmetry axis. A comparison with the silicon lens coupled PPWG shows that the metal cylinders couple energy into the $\mathrm{TE}_{1}$ mode more efficiently.

For the TE-modes, the two-cylinder coupler offers a significant improvement of performance. Figure 4 shows the comparison between using silicon lenses to couple into a 30 $\mathrm{mm}$ long PPWG and using the two-cylinder coupler, where both have the gap width of $100 \mu \mathrm{m}$. In addition to the better amplitude transmission (see inset in Fig. 4), the dispersion is considerably reduced, due to the small $1 \mathrm{~mm}$ propagation length in the central approximately constant gap region of the coupler. While the PPWG still shows oscillations of the transmitted terahertz pulse beyond $100 \mathrm{ps,} \mathrm{the} \mathrm{oscillation} \mathrm{du-}$ ration for the two-cylinder coupler is less than $50 \mathrm{ps}$. The adiabatic coupling of the two-cylinder coupler improves the amplitude transmission and also eliminates the troublesome reflection from the alternative coupling lenses.

In conclusion, we have shown that a matched pair of circular chords cut from an aluminum cylinder can be used to form an efficient broadband coupler (without any transmission optics) to an attached PPWG. An amplitude transmission of $18 \%$ was observed for the TEM-mode, while for the $\mathrm{TE}_{1}$ mode, a much higher transmission of $50 \%$ was obtained.

This work was partially supported by the National Science Foundation and the Fraunhofer Gesellschaft FhG internal program for the scientific exchange (PROF. ${ }^{2}$ ).

${ }^{1}$ J. Melinger, N. Laman, S. Sree Harsha, and D. Grischkowsky, Appl. Phys. Lett. 89, 251110 (2006).

${ }^{2}$ J. Zhang and D. Grischkowsky, Opt. Lett. 29, 1617 (2004).

${ }^{3}$ N. Laman, S. Sree Harsha, D. Grischkowsky, and J. Melinger, Opt. Express 16, 4094 (2008).

${ }^{4}$ S.-H. Kim, E. S. Lee, Y. B. Ji, and T.-I. Jeon, Opt. Express 18, 1289 (2010).

${ }^{5}$ M. Theuer, R. Beigang, and D. Grischkowsky, Appl. Phys. Lett. 96, 191110 (2010).

${ }^{6}$ M. Theuer, S. Sree Harsha, and D. Grischkowsky, J. Appl. Phys. 108, 113105 (2010).

${ }^{7}$ J. Zhang and D. Grischkowsky, Appl. Phys. Lett. 86, 061109 (2005).

${ }^{8}$ R. Mendis, V. Astley, J. Liu, and D. M. Mittleman, Appl. Phys. Lett. 95, 171113 (2009).

${ }^{9}$ E. Lee, J. Jang, S. Kim, Y. Ji, and T. Jeon, J. Korean Phys. Soc. 53, 1891 (2008).

${ }^{10}$ R. Mendis and D. Mittleman, J. Opt. Soc. Am. B 26, A6 (2009). 